\title{
CAMPUH WARNA BASA RING SESOLAHAN WAYANG WONG SAKING SEKEHA WAYANG WONG GUNA MURTI DESA TEJAKULA SANE MAMURDA KATUNDUNG ANGGADA
}

\author{
Komang Hendri Purwanata ${ }^{1}$, I.A.Sukma Wirani ${ }^{1}$, Ida Bagus Rai ${ }^{2}$ \\ Jurusan Pendidikan Bahasa Bali \\ Universitas Pendidikan Ganesha \\ Singaraja, Indonesia
}

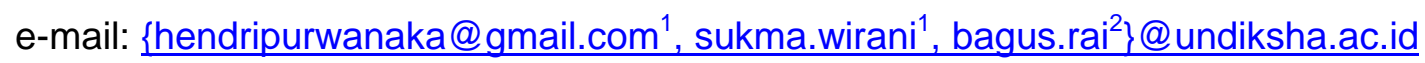

\section{Kuub}

Tetilikan puniki matetujon mahbahang (1) soroh campuh warna basa manut wangun kerta basa ring sesolahan wayang Guna Murti Desa Tejakula sane mamurda Katundung Anggada, (2) soroh campuh warna basa manut wit basa ring sesolahan wayang Guna Murti Desa Tejakula sane mamurda Katundung Anggada, (3) kahanan campuh warna basa ring sesolahan wayang Guna Murti Desa Tejakula sane mamurda Katundung Anggada, (4) pikenoh campuh warna basa ring sesolahan wayang Guna Murti Desa Tejakula sane mamurda Katundung Anggada. Jejering tetilikan inggih punika lelampahan wayang wong sane mamurda Katundung Anggada. Panandang tetilikan inggih punika campuh warna basa. Kramaning tetilikan sane kaanggen inggih punika deskriptif kualitatif minakadi transkripsi video, dokumentasi, lan sadu wicara. Data tureksa sane kaanggen sakadi reduksi data, nyorohang data, pangwedar, lan panyutetan. Pikolih tetilkan minakadi, (1) soroh campuh warna basa manut wangun kerta basa marupa morfem, frasa, lan klausa, (2) soroh campuh warna basa manut wit basa marupa campuh warna basa ka tengah, ka sisi, lan campuhan, (3) kahanan sane ngawinang campuh warna basa sakadi angge bebanyolan, keceplosan, nganutin genah, mangda sane mirengang resep, nganutin aab, nganutin pengalaman, (4) pikenoh campuh warna basa angge ngadungang bebaosan, angge ngwentenang variasi basa, angge ngresepang sang sane mirengang.

Kruna jejaton : campuh warna basa, katundung anggada, wayang wong

\begin{abstract}
Abstrak
Tujuan dari penelitian ini adalah menjelaskan (1) jenis campur kode menurut unsur-unsur bahasa dalam dialog pementasan wayang wong Guna Murti Desa Tejakula yang berjudul Katundung Anggada, (2) jenis-jenis campur kode menurut asal bahasa dalam dialog pementasan wayang wong Guna Murti Desa Tejakula yang berjudul Katundung Anggada, (3) faktor-faktor penyebab terjadinya campur kode dalam dialog pementasan wayang wong Guna Murti Desa Tejakula yang berjudul Katundung Anggada, (4) manfaat campur kode dalam dialog pementasan wayang wong Guna Murti Desa Tejakula yang berjudul Katundung Anggada. Subjek dari penelitian adalah pementasan wayang wong yang berjudul Katundung Anggada. Objek penelitian adalah campur kode. Metode penelitian yang digunakan yaitu tarnskrip video, dokumentasi, dan wawancara. Analisis data yang digunakan seperti identifikasi data, reduksi data, klasifikasi data, dan kesimpulan. Hasil penelitian yaitu, (1) jenis campur kode menurut unsur bahasa yaitu morfem, frasa, klausa, (2) jenis campur kode menurut asal bahasa, ada campur kode ke tengah, ada campur kode ke luar, dan ada campur kode campuran, (3) faktor yang menyebabkan terjadinya campur kode yaitu, untuk menampilkan kelucuan, keceplosan, menyesuaikan tempat, pendengar mengerti yang diucapkan, mengikuti zaman, sesuai pengalaman, (4) manfaat campur kode yaitu dialog agar terarah dan nyambung, agar ada variasi bahasa, agar yang mendengarkan bisa mengerti.
\end{abstract}

Kata kunci : campur kode, katundung anggada, wayang wong 


\begin{abstract}
The aims of the study are to describe the dialogue of Wayang Wong Guna Murti, Tejakula village entitles Katundung Anggada. It is focused on (1) type of code-mixing relates to the language features, (2) type of code mixing relates to the origin of the language, (3) factors that cause the code-mixing, (4) and the benefits of code-mixing itself. The subject of the study is the performance of Wayang Wong Guna Murti entitled Katundung Anggada while the object is the code-mixing itself. The video transcription, documentation, and interview are used in order to collect the data as the method. The data analysis that is used such as data identification, data reduction, data clarification, and summarization. The result of this study was found that (1) type of code-mixing relates to the language features such as morpheme, phrase, and clause, (2) type of code-mixing relates to the origin of the language such as, inner code-mixing, outer codemixing, blended code-mixing, (3) the factors that cause code-mixing such as, for showing humor, a slip of the tongue, adjusting places, understandable by the hearer, following the period, suitable experience, (4) and the benefits of code-mixing such as, to make the dialogue is going welldirected, with the variation of language, so it's understandable by the hearer.
\end{abstract}

Keywords: code-mixing, katundung anggada, wayang wong

\section{PURWAKA}

Wayang madue artos bayang utawi lawatan. Nanging yening selehin malih wayang madue artos suluh idup utawi cerminan olih para krama soang-soang. Wayang pinaka silih sinunggil kasenian sane wenten ring Indonesia sane madaging indik peplajahan (pendidikan), lan filsafat sane patut kaplajahin (Mulyono, 1989 : 9). Wayang taler kasolahang olih dalang lan wayang pinaka pirantinyane (Brandon dan Soedarsono, Seni Pertunjukan Indonesia di Era Global, 1998 : 30).

Wenten wayang sane kasolahang olih manusa, sane ngangge pangangge (kostum) rikala masolah inggih punika wayang wong. Wenten taler wayang sane ngangge kulit marupa manusa, raksasa, buron miwah sane lianan pinaka pirantinnyane inggih punika wayang kulit.

Wayang kulit punika kasolahang olih dalang ngangge kelir lan blencong (damar) taler kawantu olih katengkong (sane ngayahin dalang rikala nyolahang wayang), lan juru gender akehnyane apasang utawi duang pasang. Wayang Kulit kakepah dados 2 soroh inggih punika wayang kulit wali lan wayang kulit balih-balihan. Wayang Kulit wali minakadi : Wayang Kulit Lemah, Wayang Kulit Calonarang, lan Wayang Kulit Sapuh Leger. Taler yening Wayang Kulit balih-balihan minakadi : Wayang Kulit
Parwa, Wayang Kulit Ramayana, Wayang Kulit Cupak, Wayang Kulit Arja, lan Wayang Kulit Inovatif.

Wayang wong pinaka sesolahan drama tari sane wenten ring Indonesia. Wayang wong ring Bali sampun wenten ring abad XVI (1460-1550), rikala kasenian Bali wenten ring pamucuk masa Dalem Watu Renggong (Budi Artha, 2004 : 1). Wayang wong ring Bali pinaka sesolahan drama tari sane ngangge tapel lan ngangge basa Jawa kuna taler ngangge lelampahan wiracarita saking Ramayana lan Mahabharata (Soedarsono, 2002 : 140). Wayang wong ring Bali kakepah dados kalih inggih punika wayang wong Parwa lan wayang wong Ramayana. Sane ngabinayang wayang wong Parwa lan Ramayana inggih punika saking carita sane kaanggen. Yening wayang wong Parwa ngangge wiracarita Mahabharata, nanging yening wayang wong Ramayana nganutin sakadi parinamannyane ngangge wiracarita saking Ramayana. Asapunika taler yening wayang wong Parwa samian pragina nenten ngangge tapel sajabaning punakawan, nanging yening wayang wong Ramayana samian pragina ngangge tapel. Ngraris kawentenan wayang wong ring Bali taler sampun nglimbak nganutin aab, yening wayang wong Ramayana mangkin sampun ketah kasambatang wayang wong manten, nanging yening wayang wong 
Parwa lumbrah kasambatang Parwa manten (Bandem, 1983 : 147)

Manut Jro Dalang Made Sadnyana (tokoh seniman wayang wong Desa Tejakula) maosang, rikala para panglingsir Desa Tejakula ngripta kasenian wayang wong puniki wantah ngambil carita saking itihasa utawi carita para Raja/Ksatria sane sampun lintang, duaning dumun wantah carita punika sane nyritayang indik para Raja/Ksatria, antuk punika wayang wong wantah nganggen carita Ramayana lan Mahabaratha (Itihasa) (sadu wicara rahina Wrespati, 22 Pebruari 2018). Bandem lan Murgiyanto taler maosang, duaning wiracarita punika sampun kasub, antuk punika para seniman ngaryanang drama tari sane nyritayang indik wiracarita (Bandem, Murgiyanto, 1996 : 34).

Wayang wong prasida kabaos kasenian sane jangkep duaning madue unsur gerak, gegendingan (sendon), gamelan, lan basa. Gerak sane wenten ring kasenian wayang wong pateh sakadi gerak sane wenten ring tari-tari sane anyar, minakadi agem, tanjek, ipek capung, ngupak lantang, nayog, malpal, miwah sane lianan, nanging geraknyane kuna (klasik).

Rikala mabebaosan, samian pragina ngangge basa sane sampun kapatutang lan nganutin pakem sane wenten, inggih punika ngangge basa sane sujati, minakadi ngangge basa kawi (Jawa Kuna) sane patut lan becik asapunika taler ring basa Bali. Basa Kawi sering kabaos basa Jawa Kuna, duaning basa Jawa Kuna puniki basa sane kaangge sadina-dina rikala masa Hindu ring Jawi (Majapahit) ngraris kasobiahang ngantos ka Bali. Basa Bali inggih punika basa sane wenten lan kaangge ring Bali. Basa Bali punika kaanggen piranti mabebaosan olih krama Bali. Basa Bali mangkin sane kasengguh basa Bali Anyar (modern) sampun akeh kaiusin olih panglimbak basa sane lianan. Indik panglimbak basa Bali sadurung sakadi mangkin kakepah dados tigang panglimbak sane kawastanin aab (periodisasi) panglimbak basa Bali. Tigang pahan panglimbak punika (1) basa Bali sane akeh keni iusan kosa basa Sanskerta sane sering kasengguh basa Bali Kuna, (2) basa Bali sane kosa basanyane akeh kapanggihin kosa basa Jawa lan kosa basa
Jawa kuna sane kasengguh basa Bali Tengahan, (3) basa Bali sane kosa basanyane nenten wantah kacampuhin olih kosa basa Sanskerta lan Jawa kuna kemanten, nanging sampun akeh kapanggihin kosa basa Indonesia, Cina, taler basa dura negara sane lianan kasengguh basa Bali Anyar (modern) (Bawa dkk, 1985: 21).

Basa Bali kacampuhin antuk basa sane lianan sakadi basa Indonesia lan basa sane lianan. Pateh sakadi kawentenan sekeha wayang wong Guna Murti sane wenten ring Desa Tejakula rikala nyolahang lelampahan Katundung Anggada, ring bebaosan punakawan (Malen, Wana, Delem, Sangut) ngangge basa lianan tekening basa aslinyane. Sekeha wayang wong sane sampun wenten saking warsa 1974 punika sampun polih masolah ring Taman Mini Indonesia Indah (1980, 1984, 1989, 1993, 1995), ring dura negara minakadi ring Swedia (1995) lan Jepang (1993). Ngantos mangkin sekeha wayang wong Guna Murti Desa Tejakula kari masolah ring Art Centre Denpasar rikala wenten acara PKB (Pesta Kesenian Bali).

Sekeha wayang wong Guna Murti rikala nyolahang wayang wong, sering ngangge lelampahan sane ngambil saking wiracarita Ramayana, inggih punika pati Prahasta, pati Kumbakarna, kautus Anoman, Sita kapandung, lan katundung Anggada. Carita katundung anggada boya saking carita Ramayana, sakemaon wantah kawi-kawian para dalang. Carita katundung Anggada puniki nyritayang rikala sang Anggada kapisuna olih sang Jembawan angremih Dyah Trijata, ngraris katundung olih sang Sugriwa. Sesolahan wayang wong sane mamurda Katundung Anggada puniki kari ngangge pakem gerak asli lan basa kawi sajabaning para punakawan minakadi Tualen, Wana (yening ring Bali Selatan Mredah), Delem, lan Sangut ngangge basa Bali. Punakawan puniki sane negesin sang para ratu rikala mabebaosan ngangge basa Kawi. Antuk punika para punakawan puniki rikala negesin punika mangda ngangge basa Bali sane elah karesepang olih para pamiarsa (penonton). Antuk kawentenan jagat sane kabaos aor tan pawates, akeh kapanggihin kruna-kruna saking dura Bali sajeroning dialog punika, 
minakadi madaging basa Indonesia. Kosa basa saking basa Indonesia sane sering kapiarsayang ring dialog wayang wong marupa kosa basa sane ketah kaanggen tur kauningin olih krama Bali. Kosa basa sane mawit saking basa dura Bali, nanging wenten ring bebaosan krama Bali sane marupa lengkara basa Bali punika kabaos campuh warna basa (Suhardi, 2009). Campuh warna basa wantah wangun basa lianan sane ngranjing ritatkala mabebaosan nganggen basa sane sampun kapastiang (Andyka, 2013). Ketahnyane, campuh warna basa punika medal riantukan parisolah sang sane mabaos, sakadi wewidangan pakraman, kawagedan mabasa, miwah pangrasa maagama. Lianan ring punika, karumaketan sang sane mabaos, genah miwah galah (situasi) informal, miwah katunaan basa utawi nenten wentennyane basa sane manut kaanggen, dados parindikan sane prasida ngamedalang campuh warna basa (Nababan, 1991). Sakadi sane sampun katelatarang ring ajeng, indike punika nyihnayang kawentenan campuh warna basa ring sesolahan wayang wong puniki. Yening indike ring ajeng nglantur mamargi sakadi punika, pastika wayang wong nenten prasida nglestariang basa Bali (nganutin pakem) riantukan akeh krunakruna ring basa Bali ical tur kagentosin antuk kruna-kruna sane kaambil saking basa dura Bali. Antuk kawentenan punika, patut kalaksanayang tetilik nganutin indik campuh warna basa ring wayang wong. Tetilik sane kalaksanayang pacang nilikin silih sinunggil sesolahan wayang wong saking sekeha wayang wong Guna Murti Desa Tejakula sane mamurda Katundung Anggada.

Maduluran antuk panelataran sakadi ring ajeng, tetilikan punika ngunggahang indik campuh warna basa ring sesolahan wayang wong saking sekeha wayang wong Guna Murti Desa Tejakula sane mamurda Katundung Anggada. Tetilikan puniki nelatarang indik soroh miwah dadalan campuh warna basa sane wenten ring sesolahan wayang wong saking sekeha wayang wong Guna Murti Desa Tejakula sane mamurda Katundung Anggada.

Bantang pikobet inggih punika (1) kawentenan campuh warna basa sajeroning wangun kerta basa ring sesolahan wayang wong Guna Murti Desa Tejakula sane mamurda Katundung Anggada, (2) wangun campuh warna basa sajeroning wit basa ring sesolahan wayang wong Guna Murti Desa Tejakula sane mamurda Katundung Anggada, (3) kahanan sane ngawinang wenten campuh warna basa ring sesolahan wayang wong Guna Murti Desa Tejakula sane mamurda Katundung Anggada, (4) pikenoh campuh warna basa ring sesolahan wayang wong Guna Murti Desa Tejakula sane mamurda Katundung Anggada.

\section{KRAMANING TETILIK}

Pendekatan lan soroh tetilikan sane kanggen ring tetilikan puniki inggih punika tetilikan deskriptif kualitatif santukan matetujon mahbahang wangun campuh warna basa ring lelampahan wayang wong sane mamurda Katundung Anggada olih sekeha wayang wong Guna Murti Desa Tejakula. Tetilikan deskriptif kualitatif mautsaha nyihnayang kasujatian (fakta) utawi fenomena sane wenten ri kala tetilikan punika memargi antuk ngunggahang napi sane sapatutnyane wenten. Ring tetilikan puniki panilik ngambil data antuk ngemargiang tata cara mupulang data sane kaadungang sareng data tureksanyane. Lianan ring punika pendekatan deskriptif kualitatif kanggen mangda ngamolihang data sane patut manut kadi kasujatian saking data sane katilikin. Genah tetilikan puniki ring kesekretariatan sekeha wayang wong Guna Murti (Balai Desa Tejakula) Desa Tejakula, Kecamatan Tejakula, Kabupaten Buleleng.

Wit data sajeroning tetilikan puniki inggih punika panilik ngrereh video wayang wong saking sekeha wayang wong Guna Murti Desa Tejakula sane mamurda Katundung Anggada sane masolah ring Swedia warsa 1995. Parikrama mupulang data inggih punika tata cara sane kaanggen sajeroning ngrereh data antuk sasuratan miwah bebaosan. Tetujon mupulang data inggih punika mangda ngamolihang data sane kaaptiang. Wenten makudang-kudang pah-pahan rikala ngamargiang parikrama mupulang data inggih punika kramaning 
transkripsi, piranti dokumentasi, lan piranti sadu wicara.

Data tureksa puniki kamargiang ri sampun data punika kapolihang. Tata cara ngolah data kalaksanayang antuk nureksain data nganggen kramaning deskriptif kualitatif. Tureksa data puniki kalaksanayag antuk nyritayang utawi nelatarang data sane sampun kapolihang. Ngenenin indik pamargi data tureksa sane kalaksanayang ring sajeroning tetilik puniki katelatarang sakadi ring sor inggih punika wenten reduksi data, nyorohang data, pangwedar data, lan panyutetan.

\section{PIKOLIH LAN TETEPASAN}

Sajeroning adyaya puniki pacang mahbahang ngenenin indik pikolih lan tetepasan tetilikan saking 4 bantang pikobet inggih punika (1) kawentenan campuh warna basa ring sesolahan wayang wong Guna Murti Desa Tejakula sane mamurda Katundung Anggada, (2) wangun campuh warna basa ring sesolahan wayang wong Guna Murti Desa Tejakula sane mamurda Katundung Anggada, (3) kahanan sane ngawinang wenten campuh warna basa ring sesolahan wayang wong Guna Murti Desa Tejakula sane mamurda Katundung Anggada, (4) pikenoh campuh warna basa ring sesolahan wayang wong Guna Murti Desa Tejakula sane mamurda Katundung Anggada

1. Kawentenan Campuh Warna Basa Ring Sesolahan Wayang Wong Guna Murti Desa Tejakula Sane Mamurda Katundung Anggada Manut Wangun Kerta Basa

Wenten makudang-kudang data sane kapolihang ring tetilikin puniki. Yening nganutin pakem, wenten 2 soroh basa sane kaanggen rikala wayang wong masolah, inggih punika wenten basa Jawa Kuna (kaanggen olih para ratu), basa Bali (kaanggen olih para punakawan). Sakemaon ring sesolahan puniki nenten nganutin pakem, akeh basa lianan sane ngranjing ring sesolahan puniki, wenten basa Indonesia lan basa Inggris. Wenten dialog punakawan sane ngangge basa Bali kacampuhin ngangge basa Jawa Kuna, yening indike punika nenten rumasuk campuh warna basa nganutin wangun kerta basa, duaning basa Jawa Kuna lan basa Bali pinaka basa utama rikala nyolahang wayang wong. Napi sane ngawinang wayang wong punika wantah ngangge basa Jawa Kuna lan basa Bali (nganutin pakem), duaning manut Jro Dalang Made Sadnyana (sadu wicara rahina Radite, 3 Juni 2018) wayang wong ngambil carita saking kekawin Ramayana utawi Mahabaratha, ring kekawin punika ngangge basa Jawa Kuna. Kawentenan wayang wong ring Bali kirang langkung duk abad ka-16, duaning wayang wong punika kasub ring Bali, pragina ratu ngangge basa Jawa Kuna (basa nganutin kekawin) ngraris basa Bali kaanggen negesin sajeroning basa Jawa Kuna sane kaanggen olih para Ratu.

Sajeroning tetilikan puniki kapolihang data akehnyane 30 sane marupa campuh warna basa manut wangun kerta basa. Data-data punika kakepah dados 4 inggih punika wangun kerta basa sane rumasuk ring morfem bebas akeh data 17, morfem terikat akeh data 9, frasa akeh data 3, lan klausa akeh data 1. Data campuh warna basa manut wangun kerta basa sane marupa morfem punika kruna sane kawangun praragan lan sampun madue arti (Gorys Keraf ring Kridalaksana, 1993:25).

Tetepasan data campuh warna basa ring klausa molihang siki data wenten lari tunggang langgang. Mawinan "lari tunggang langgang" punika ngranjing ring klausa duaning kruna "lari" rumasuk jejering lan "tunggang langgang" rumasuk linging, taler Chaer (1994:231) maosang klausa punika angkepan kruna sane sakirang-kirangnyane kawangun antuk jejering miwah linging.

\section{Wangun Campuh Warna Basa Ring Sesolahan Wayang Wong Guna Murti Desa Tejakula Sane Mamurda Katundung Anggada}

Campuh warna basa manut wit basa kakepah dados 3 inggih punika campuh warna basa ka tengah, campuh warna basa ka sisi, lan campuh warna basa campuhan (Suwito, 1996:89). Pikolih sane kapertama campuh warna basa ka tengah molihang data 29 minakadi selamat, tolak, banyak, omong, marah, selingkuh, sadar, setia, menurut, kumpul, takdir, semangat, sehat, capek, semakin, tangkapan, kosong, 
cobak, berpikir, terbayang, balas dendam, lari, tunggang langgang. Pikolih data ring ajeng kasengguh campuh warna basa ka tengah, punika soroh campuh kode sane ngranjing ring pawiwit basa asli sane kantun asoroh (serumpun).

Kaping kalih indik campuh warna basa kasisi sane nenten sarumpun. Wenten 1 kruna sane rumasuk campuh warna basa ka sisi inggih punika kruna full.

Campuh warna basa ka sisi molihang data wantah 1. Campuh warna basa campuhan inggih punika campuh warna basa sane ngranjingang wangunwangun basa sane asoroh (serumpun) miwah basa sane nenten saking asoroh. Kawentenan campuh warna basa campuhan, duaning basa ring dialog wayang wong punika madaging basa utama (basa Bali) taler wenten basa sane lianan sane sarumpun (basa Indonesia lan Jawa Kuna) lan madaging basa sane nenten sarumpun sareng basa utama (basa Sanskerta lan basa Inggris), sakadi imba ring dialog "bi bojog gede ne Ngut, tumben bli mencar maan bojog Tut, maan kera, maan monkey Ngut". Kruna kera punika wit basa saking basa Indonesia lan basa Indonesia serumpun sareng basa Bali (austronesia). Ngraris kruna monkey wit saking basa Inggris lan basa Inggris punika nenten serumpun sareng basa utama (basa Bali). Duaning wenten campuhan warna basa serumpun (ka tengah) lan wenten campuh warna basa nente serumpunn (ka sisi) antuk punika wenten campuh warna basa campuhan ring dialog wayang wong punika.

\section{Kahanan Sane Ngawinang Wenten Campuh Warna Basa Ring Sesolahan Wayang Wong Guna Murti Desa Tejakula Sane Mamurda Katundung Anggada}

Kawentenan campuh warna basa ring sesolahan wayang wong Guna Murti Desa Tejakula sane mamurda Katundung Anggada akeh kapanggihin bebaosan para pragina wayang wong. Parindikan punika medal saking sang sane mabaos utawi pragina wayang wong punika. Pikolih data indik kahanan sane ngawinang wenten campuh warna basa ring sesolahan wayang wong puniki antuk kramaning sadu wicara majeng ring makudang-kudang pragina wayang wong punika utaminnyane para pragina punakawan sane akeh madaging campuhan ring bebaosannyane sane kalaksanayang ring Desa Tejakula Kecamatan Tejakula Kabupaten Buleleng. kramaning sadu wicara puniki kamargiang ngawit saking rahina Soma, 21 Mei 2018 ngantos rahina Sukra, 8 Juni 2018. Saking kramaning sadu wicara sane sampun kalaksanayang saking 9 pragina wayang wong inggih punika wenten Jro Dalang Made Sadnyana (Malen), Ketut Mulya (Wana), Gede Putu Tirta Ngis (Delem), Gede Suparta (Sangut), Gede Puja Adnyana (Sugriwa), Nyoman Nuarta (Jembawan), Nyoman Artaya (Hanoman), Ketut Widiasa (Anggada), Gede Sugiantara (Durga). Wenten makudang-kudang panampen sane kapolihang, wenten panampen sane pateh lan malianan. Yening kacutetang wenten 9 data inggih punika, (1) duaning keceplosan, (2) nganutin genah masolah, (3) mangda penonton resep, (4) duaning basa Bali sane kabaosang meweh kagentosin antuk basa lianan, (5) angge bebanyolan, (6) sampun terbiasa mabasa campuhan, (7) nganutin aab sane sampun nglimbak, (8) nganutin pengalaman pragina punika, (9) nganutin sane kaajak mabebaosan. Yening saihang sareng teori sane wenten ring sepat sikusiku wenten kalih sane ngawinang wenten campuh warna basa inggih punika parindikan saking sang sane mabaos lan parindikan basa. Yening kacingak sareng data sane kapolihang sane ngranjing ring parindikan saking sang sane mabaos inggih punika: 1) nganutin genah masolah, 2) mangda panonton resep, 3) angge bebanyolan, 4) duaning basa Bali sane kabaosang meweh kagentosin antuk basa lianan, 5) nganutin aab sane sampun nglimbak, 6) nganutin pengalaman pragina punika. Taler manut parindikan basa inggih punika: 1) duaning keceplosan, 2) sampun sarahina ngangge basa campuhan, 3) nganutin sane kaajak mabebaosan.

4. Pikenoh Campuh Warna Basa ring Sesolahan Wayang Wong Guna Murti Desa Tejakula Sane Mamurda Katundung Anggada.

Kawentenan campuh warna basa ring sesolahan wayang Guna Murti Desa Tejakula sane mamurda Katundung 
Anggada nenten lempas saking pikenoh majeng ring pragina rikala mabebaosan nyolahang wayang wong puniki. Mangda prasida ngamolihang data pikenoh campuh warna bsa ring sesolahan wayang wong Guna Murti Desa Tejakula sane mamurda Katundung Anggada puniki, panilik ngawentenang sadu wicara majeng ring pragina wayang wong. pikolih data indik pikenoh campuh warna basa ring sesolahan wayang wong Guna Murti Desa Tejakula sane mamurda Katundung Anggada pateh sakadi data parikrama sadu wicara sane kalaksanayang ring ajeng.

Saking kramaning saduwicara sane sampun kamargiang, wenten makudangkudang data sane kapolihang indik pikenoh basa campuh ring sesolahan wayang wong sekeha Guna Murti Desa Tejakula sane mamurda "katundung anggada" puniki. Wenten pasaur sane malianan taler wenten pasaur sane pateh saking narawakia. Wenten 4 data sane kapolihang inggih punika (1) bebaosane mangda adung, yadiastun bebaosane ring dialog wayang punika madaging basa campuhan sakemaon yening para pragina sane mabebaosan punika sampun resep olih sane mirengang, punika bebaosan sane becik, nanging yening sane mabebaosan ngangge basa Bali sakemaon sane mirengang (lawan tutur) nenten resep nika ngawinang bebaosane nenten adung (tidak efektif).

(2) angge bebanyolan, ring panglimbak aab sakadi mangkin bebanyolan pinaka sinalih tunggil sane patut madaging ring sesolahan-sesolahan (balih-balihan) mangda sesolahane nenten tegang, asapunika sakadi sesolahan wayang wong.

(3) wenten variasi basa, asapunika taler ring aab sakadi mangkin variasi basa punika mabuat pisan kaplajahin duaning mangda nguningin kosa basa utawi arti-arti anyar (istilah baru) ring dialog, antuk punika bebaosane mangda adung. Kawentenan variasi basa punika duaning status sosial sang sane mabebaosan irika, napike faktor pendidikan, pengalaman, miwah sane lianan, antuk punika variasi basa ring dialog wayang wong pastika wenten, ngraris pragina utawi lawan bicara sane durung nguningin istilah anyar punika dados uning duaning lawan bicara punika ngangge istilah anyar.

(4) sane mirengang resep, sane pinih utama rikala nyolahang sesolahan punika mangda panonton resep, mangda panontone uning ring carita sane kasolahang, antuk punika patut yening bebaosane kadagingin basa campuhan.

\section{Implikasi}

Sakadi sane sampun kabahbahang ring tetilikan asoroh, tetilikan indik wayang wong sadurungnyane sampun polih katilikin olih Ari Widana (2016). Nanging ring tetilikan puniki malianan sareng tetilikan saking Widana. Tetilikan Widana maosang indik kawentenan tapel, gelungan, miwah busana sane kaanggen olih pragina wayang wong Desa Tejakula. Tetilikan Widana punika maosang indik kawentenan tapel wayang wong minakadi wangun tapel wayang wong punika (ukuran tapel), asapunika taler ring kawentenan gelungan soang-soang tapel punika, sakadi wenten gelung tajug, gelung apit surang, gelung candi kurung miwah sane lianan, taler kawentenan busana sane kaange olih soang-soang pragina wayang wong.

Malianan sareng tetilikane puniki inggih punika nilikin indik kawentenan basa sane wenten ring wayang wong Desa Tejakula sane mamurda Katundung Anggada. Sajeroning kawentenan campuh warna basa ring tetilikan puniki ngenenin indik wangun kerta basa (morfem, frasa, klausa), lan wangun campuh warna basa (campuh warna basa ka tengah, campuh warna basa kasisi, campuh warna basa campuhan).

Wesana campuh warna basa ring sesolahan wayang wong Desa Tejakula inggih punika yening kacingak saking panglimbak aab sakadi mangkin becik pisan duaning mangda penontos resep, taler upami genah rikala nyolahang wayang wong punika ring Jakarta nenten prasida antuka ngangge basa Bali manten, duaning panontone wenten saking dura Bali, asapunika taler masolah ring luar negeri pastika ngangge basa Inggris utawi sane lianan. sakemaon yening cingakin nganutin pakem pastika nenten nganutin pakem sane wenten ring wayang wong. Yening 
rikala masolah akehan ngangge basa Indonesia utawi basa lianan, kasuen-suen para pragina nenten malih nyingakin pakem rikala masolah lan para pragina ngangge basa Indonesia rikala mabebaosan nenten malih ngangge basa Bali lan basa Jawa Kuna.

Pikenoh tetilikan puniki mangda kawentenan wayang wong ring Desa Tejakula stata nganutin pakem sane wenten minakadi ring bebaosan para pragina utaminnyane para punakawan, duaning akeh kapanggihin basa saking dura Bali sane ngranjing ring dialog wayang wong puniki. Kaaptiang majeng ring pragina wayang wong Desa Tejakula mangda tetilikan puniki kaanggen tetimbang lan mangda nguningin yening akeh kosa basa saking basa lianan tekening basa Bali ngranjing ring dialog wayang wong puniki. Antuk punika para pragina wayang wong ring Desa Tejakula mangda malajahin malih kosa basa Bali sane patut, mangda wayang wong Desa Tejakula stata nganutin pakem rikala masolah.

\section{PAMUPUT}

Tetingkesan

Saking pikolih lan tetepasan sane sampun kabahbahang ring ajeng, prasida katingkesang sakadi ring sor.

1. Campuh warna basa manut wangun kerta basa sajeroning bebaosan pragina wayang wong Desa Tejakula sane mamurda Katundung Anggada kapolihang 30 data. Ngraris data punika kakepah dados 4 inggih punika nganutin morfem bebas, morfem terikat, frasa, lan klausa. Data sane marupa morfem bebas wenten 17 data inggih punika : selamat, tolak, marah, nyelingkuhin, selingkuh, sadar, setia, menurut, ngrayu, kumpul, takdir, semangat, sehat, nyemangatang, capek, semakin, tangkapan, kosong, cobak, full, kera, monkey, berpikir, terbayang. Data manut morfem terikat wenten 9 data inggih punika : nyelingkuhin, menurut, ngrayu, nyemangatang, semakin, tangkapan, berpikir, terbayang. Data manut frasa wenten 4 data inggih punika : banyak bicara, terus semangat, balas dendam, tak kasatmata. Data manut klausa wenten siki data inggih punika lari tunggang langgang. 2. Campuh warna basa manut wit basa sajeroning bebaosan pragina wayang wong Desa Tejakula sane mamurda Katundung Anggada kapolihang 31 data inggih punika 29 data saking campuh warna basa ka tengah inggih punika wenten kruna selamat, tolak, banyak, omong, marah, nyelingkuhin, selingkuh, sadar, setia, menurut, ngrayu, kumpul, takdir, semangat, sehat, nyamangatang, capek, semakin, tangkapan, kosong, cobak, berpikir, terbayang, balas dendam, lari tunggang langgang. Ngraris campuh warna basa kasisi wenten 1 data inggih punika kruna full. Sane kaping untat campuh warna basa campuhan wenten siki data inggih punika ring lengkara "Bi bojog gede ne Ngut, tumben bli mencar maan bojog Tut, maan kera, maan monkey ngut".

3. Kahanan sane ngawinang wenten campuh warna basa ring sesolahan wayang wong Guna Murti Desa Tejakula sane mamurda Katundung Anggada. Manut sepat siku-siku sane sampun kaanutang sareng pikolih saadu wicara sareng narawakya kapolihang tetingkesan sane ngwesanain campuh warn basa ring sesolahan wayang wong Guna Murti Desa Tejakula sane mamurda Katundung Anggada inggih punika (1) duaningg keceplosan, (2) nganutin genah masolah, (3) mangda penonton resep, (4) duaning basa Bali sane kabaosang meweh kagentosin antuk basa lianan, (5) angge bebanyolan, (6) sampun terbiasa mabasa campuhan, (7) nganutin aab sane sampun nglimbak, (8) nganutin pengalaman pragina punika, (9) nganutin sane kaajak mabebaosan.

4. Pikenoh campuh warna basa ring sesolahan wayang wong Guna Murti Desa Tejakula sane mamurda Katundung Anggada. Manut sadu wicara sane kalaksanayang majeng ring narawakia kapolihang makudang-kudang panampen manut pragina wayang wong inggih punika (1) bebaosane mangda adung, (2) angge bebanyolan, (3) wenten variasi basa, lan (4) sane mirengang mangda resep. 


\section{Piteket}

Malarapan antuk sane sampun kabahbahang, wentek piteket-piteket sane jagi kabahbahang sakadi ring sor.

1. Majeng ring panilik kaaptiang pikolih tetilikan puniki mangda prasida ngabinayang ngenenin indik wangun campuh warna basa sane wenten ring sajeroning lelampahan wayang wong taler nguningin kahanan sane ngawinang kawentenan campuh warna basa lan nguningin pikenoh campuh warna basa ring sajeroning bebaosan wayang wong.

2. Majeng ring panilik sane lianan kaaptiang mangda prasida nglimbakang tetilikan puniki pamekas nglanturang tetilikan ring wayang wong ring desa lianan tekeningg Desa Tejakula. Pangllimbak sane kaaptiang inggih punika mangda nglaksanayang tetilikan sane asoroh utawi lianan, sinalih tunggilnyane ngenenin indik campuh warna basa ring bebaosan wayang wong sane wenten ring Bali. indike punika kaaptiang kalaksanayang mangda wenten sandingan pikolih tetilikan sajeroning bebaosan wayang wong sane wenten ring Bali, duaning bebaosan soang-soang genah sane wenten kasenian wayang wong malianan.

3. Majeng ring para mahasisia kaaptiang mangda prasida kaanggen nincapang kawagedan paplajahan ring mata kuliah sosiolinguistik. Mangda mahasisia punika uning tur ngresepang indik parindikan sane ngawinang kawentenan campuh warna basa.

4. Majeng ring para pangwacen pamekas krama Bali kaaptiang mangda prasida uning indik tetamian kesenian sane sampun kuna puniki

\section{KAPUSTAKAN}

Andyka Putra Gotama, Putu. 2013. "Campur Kode dalam Pembelajaran Bahasa Bali". Lampuhyang, Volume 4(halaman 106-117).

Bandem, I Made, dan Sal Murgiyanto. 1996. Teater Daerah Indonesia. Yogyakarta: Yayasan Kanisius,
1983.Ensiklopedi Tari Bali.

Denpasar : Akademi Seni Tari Indonesia.

Bawa, I Wayan. 2002. Sejarah Perkembangan Bahasa Bali. Denpasar: Penerbit Universitas Udayana.

Budi Artha, I Nyoman. 2004. "Perkembangan Dramatari Wayang Wong Tejakula dalam Perubahan Budaya Bali”. Tesis untuk memperoleh Gelar Magister pada Program Studi Kajian Budaya Program Pascasarjana Universitas Udayana Denpasar.

Chaer, Abdul. 1994. Linguistik Umum. Jakarta: Rineka Cipta.

Kridalaksana, Harimurti. 1993. Kamus Linguistik. Jakarta : Gramedia.

Nababan, 1991. Sosial Linguistik Suatu Pengantar. Jakarta: PT Gramedia Pustaka Utama.

Soedarsono, 2002. Seni Pertunjukan Indonesia di Era Globalisasi. Yogyakarta : Gadjah Mada University Press.

Suwito. 1985. Pengantar Awal Sosiolinguistik: Teori dan Problema. Surakarta: Henari Cipta. 